\title{
Oscillation of a mountain root structure due to a Rayleigh wave incidence
}

\author{
Mitsuru Yoshida \\ Earthquake Research Institute, University of Tokyo, 1-1-1 Yayoi, Bunkyo-ku, Tokyo 113-0032, Japan
}

(Received February 23, 2001; Revised September 5, 2001; Accepted September 6, 2001)

\begin{abstract}
Spectral amplitudes of Rayleigh waves across a mountain root in the continental crust were studied by the use of the finite difference technique. The crust has a mountain root structure like that of the Tien Shan in China, and a maximum thickness of about $50 \mathrm{~km}$. The waves are numerically simulated by implementing a plane Rayleigh wave incidence on the front of the mountain root structure. The spectral amplitudes of the vertical (W) component are shown to be strongly amplified by the mountain for periods from 20 to $50 \mathrm{~s}$, with a maximum of about $7 \%$, whereas those of the radial (U) component show a slight increase with an increase in period. For the mountain structure with small-scale low velocity zones (LVZ) in the bottom of the root and in the upper mantle, amplitudes of the Wcomponent are enriched at periods slightly longer than those for the structure without the LVZ. For the mountain structure with a double large-scale LVZ in the lower crust and the upper mantle, amplitudes of the W-component are extensively amplified over a wide range beyond $50 \mathrm{~s}$. The period range from 20 to $50 \mathrm{~s}$ with high amplifications of the W-component for the mountain root structure with or without the LVZ is consistent with periods, in which the Rayleigh to Love wave conversion is dominant in observed surface waves across the Tien Shan mountains (Pedersen et al., 1998).
\end{abstract}

\section{Introduction}

Interconversion between Rayleigh waves and Love waves is likely to occur when surface waves travel over inhomogeneous crustal and upper mantle structures that are dented, inclined, or curved, as seen in large-scale mountain roots or subduction zones of the oceanic plate. A horizontal inhomogeneity in media is a plausible factor in the emergence of the surface wave conversion. When anomalous phases or unusual amplitudes on surface waves are observed (e.g., Tanimoto, 1990; Levshin et al., 1992; Kobayashi et al., 1997; Pedersen et al., 1998), they have been interpreted by horizontally inhomogeneous structures consisting of isotropic or anisotropic media along the path, or by tectonic release in the case of nuclear explosions (Press and Archambeau, 1962; Archambeau and Sammis, 1970; Aki and Tsai, 1972; Masse, 1981; Harkrider et al., 1994). Analytical or numerical studies to determine the substantial property of interconversion of surface waves have been carried out using several models, such as a graben structure (Kennett, 1984), an Alpine-like crustal structure (Snieder, 1986), a two-layer, low-velocity plug embedded within a three-layer, stratified half-space (Bostok, 1991), a cylinderlike scatterer in the crust (Friedrich et al., 1993), and a couple of dented crustal structures (Yoshida, 1996). However, information on spectral amplitudes of surface waves across a laterally inhomogeneous earth has not yet been sufficiently obtained.

In order to elucidate the basic properties of Rayleigh wave propagation over realistic mountain root structures, the

Copy right (C) The Society of Geomagnetism and Earth, Planetary and Space Sciences (SGEPSS); The Seismological Society of Japan; The Volcanological Society of Japan; The Geodetic Society of Japan; The Japanese Society for Planetary Sciences. present paper investigated the characteristics of amplitude responses of Rayleigh waves that traveled the Tien Shanlike mountain root structure. The Tien Shan is a candidate region where the conversion from Rayleigh to Love waves occurs (Pedersen et al., 1998); the Rayleigh waves are excited by nuclear explosions at Lop Nor, a Chinese test site. The present modeling analysis is performed by the use of the two-dimensional (2-D) finite difference method.

\section{Equations of Motion and Numerical Models}

The equation for P-SV motion in a medium with Lame's parameter $\lambda$, rigidity $\mu$, and density $\rho$, all of which are functions of functions of both $x$ and $z$, are:

$$
\begin{aligned}
\rho \frac{\partial^{2} u}{\partial t^{2}}= & \frac{\partial}{\partial x}\left\{\lambda\left(\frac{\partial u}{\partial x}+\frac{\partial w}{\partial z}\right)+2 \mu \frac{\partial u}{\partial x}\right\} \\
& +\frac{\partial}{\partial z}\left\{\mu\left(\frac{\partial w}{\partial x}+\frac{\partial u}{\partial z}\right)\right\}
\end{aligned}
$$

and

$$
\begin{aligned}
\rho \frac{\partial^{2} w}{\partial t^{2}}= & \frac{\partial}{\partial z}\left\{\lambda\left(\frac{\partial u}{\partial x}+\frac{\partial w}{\partial z}\right)+2 \mu \frac{\partial w}{\partial z}\right\} \\
& +\frac{\partial}{\partial x}\left\{\mu\left(\frac{\partial w}{\partial x}+\frac{\partial u}{\partial z}\right)\right\}
\end{aligned}
$$

where $u$ and $w$ are the displacement components in $x$ and $z$ directions, respectively. The material properties are discretized with a uniform spatial increment $\Delta h(=\Delta x=$ $\Delta z=5 \mathrm{~km})$ in both $x$ and $z$ directions. The boundary conditions of the tangential ( $\widehat{x z}$ and $\widehat{z x}$ ) and normal $(\widehat{z z})$ stressfree state at a surface $z=0$ are given in the following formula: 


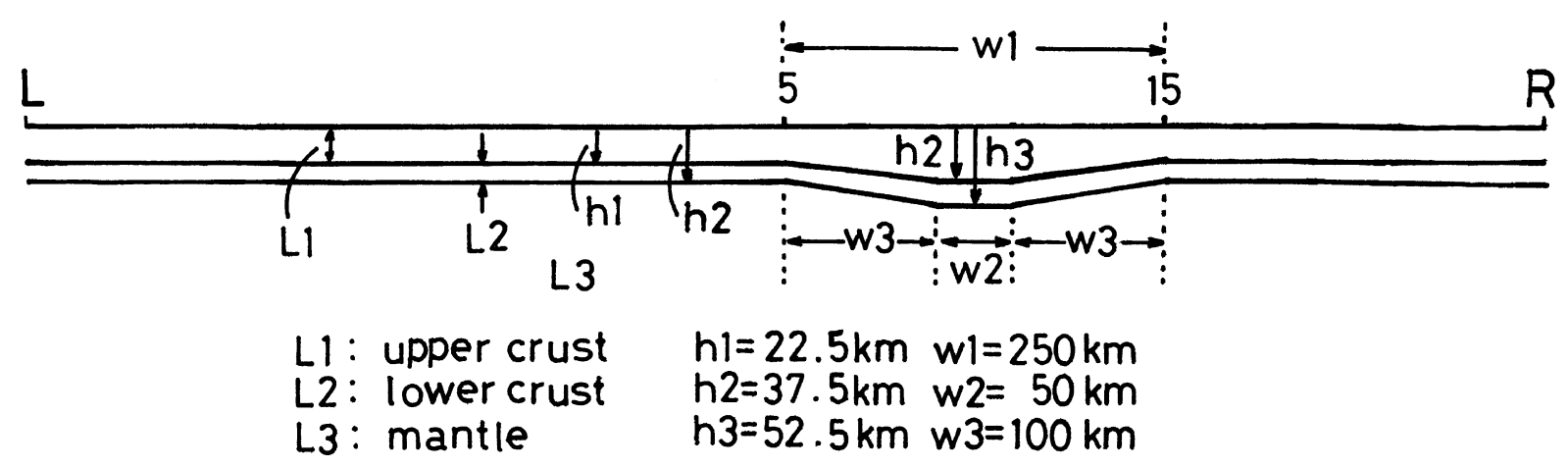

Fig. 1. Model configuration. Station sites 5 and 15 are indicated on the surface. The width ( $w 1)$ of a mountain root is assumed to be 250 km, the horizontal lengths of sloping layer $(w 3)$ and bottom $(w 2) 100$ and $50 \mathrm{~km}$, respectively. The depths of upper $(L 1)$ and lower $(L 2)$ crusts extend to $22.5(h 1)$ and $37.5(h 2) \mathrm{km}$, respectively, in the stratified medium. For the crustal model with a low velocity zone (LVZ), the LVZ is located at a depth range from 37.5 $(h 2)$ to $52.5(h 3) \mathrm{km}$ at the bottom of the mountain root (w2). Rayleigh waves are incident on the front (site 5) of the mountain and propagate towards the right. Simulated waves are observed at site 15 , which is located at the edge of the mountain root at the wave's retreat. Symbols L and R are denoted at the left and right edges of the computational space.

$$
\begin{aligned}
& \widehat{x z}=\widehat{z x}=\mu(\partial u / \partial z+\partial w / \partial x)=0 \\
& \widehat{z z}=\lambda \partial u / \partial x+(\lambda+2 \mu) \partial w / \partial z=0 .
\end{aligned}
$$

The finite difference scheme is applied to the Eqs. (1), (2), (3), and (4). The time increment $\Delta t(=0.5 \mathrm{~s})$ satisfies the stability condition (Levander, 1985)

$$
\Delta t<\Delta h / \sqrt{\alpha^{2}+\beta^{2}},
$$

where $\alpha$ and $\beta$ are the maximum compressional and shear wave velocities:

$$
\alpha=\sqrt{(\lambda+2 \mu) / \rho}
$$

and

$$
\beta=\sqrt{\mu / \rho}
$$

The A1 absorption boundary condition (Clayton and Engquist, 1977), a zero order paraxial approximation for artificial reflection, was applied at the computational sides and bottom. The spatial increment above satisfies the condition to avoid the grid dispersion; the spatial increment must be less than one-tenth of the wave length (Aki and Richard, 1980). Rayleigh wave propagation is studied as a sourcefree problem. Analysis of a problem with a source is inappropriate for the present study because the problems pertinent to a seperation of incident Rayleigh waves from reflected or converted waves and a choice of the time window for extracting incident Rayleigh wave surely arise. These problems lower the precision of the computational results, whereas, a source-free problem can treat the pure incidence of Rayleigh waves. Thus, we can obtain enough information on Rayleigh wave propagation over the mountain root with higher precision.

The mountain root model is shown in Fig. 1. This model is isotropic and consists of upper and lower crusts overlying a homogeneous mantle. The maximum depth of the dent is $52.5 \mathrm{~km}$. The elastic parameters of the model are shown in Table 1. This crustal model is similar to that of the Tien Shan mountains (Kosarev et al., 1993) located at the
Table 1. Elastic parameters of $\mathrm{P}(V p)$ and $\mathrm{S}(V S)$ waves and density $(\rho)$ used in the numerical simulations. Poisson ratio $(\sigma)$ is also shown.

\begin{tabular}{lcccc}
\hline & $\begin{array}{c}V p \\
(\mathrm{~km} / \mathrm{s})\end{array}$ & $\begin{array}{c}V s \\
(\mathrm{~km} / \mathrm{s})\end{array}$ & $\begin{array}{c}\rho \\
\left(\mathrm{Mg} / \mathrm{m}^{3}\right)\end{array}$ & $\sigma$ \\
\hline Upper crust & 6.5 & 3.9 & 2.60 & 0.22 \\
Lower crust & 7.0 & 4.0 & 2.75 & 0.26 \\
$($ LVZ $)$ & 6.5 & 3.5 & 2.60 & 0.30 \\
Mantle & 7.9 & 4.9 & 3.35 & 0.19 \\
\hline
\end{tabular}

northwestern edge of the Tarim Basin. The uniform and non-uniform wave guides are located in the left and right sides in the computational space, respectively (Fig. 1). The computational space has a horizontal length ( $x$-axis) of 1000 $\mathrm{km}$ and a vertical length (z-axis) of $600 \mathrm{~km}$. The total number of grids in the horizontal and vertical directions is 201 and 122, respectively. Site 5 is located at the midpoint between the left $(\mathrm{L})$ and right $(\mathrm{R})$ edges in the computational space. A plane Rayleigh wave is incident upon the front of the mountain root from the left. Initial values are given by

$$
\begin{aligned}
& (w)_{t=0}=h(x, z) \\
& (\partial w / \partial t)_{t=0}=i(x, z)
\end{aligned}
$$

for the $w$-component, and

$$
\begin{aligned}
& (u)_{t=0}=j(x, z) \\
& (\partial u / \partial t)_{t=0}=k(x, z)
\end{aligned}
$$

for the $u$-component, where $h(x, z), i(x, z), j(x, z)$, and $k(x, z)$ are determined from normal mode solutions for the stratified medium shown in Fig. 1 (Yoshida, 1984). The initial values, determined for spheroidal oscillations (Saito, 1967) assuming a dip slip point source and a step function in time as a seismic source, were calculated for the space domain between the left edge L and site 5 (Fig. 1), corresponding to an epicentral distance of 100 and $600 \mathrm{~km}$, respectively. The seismic moment was assumed to be $1.0+$ E26 

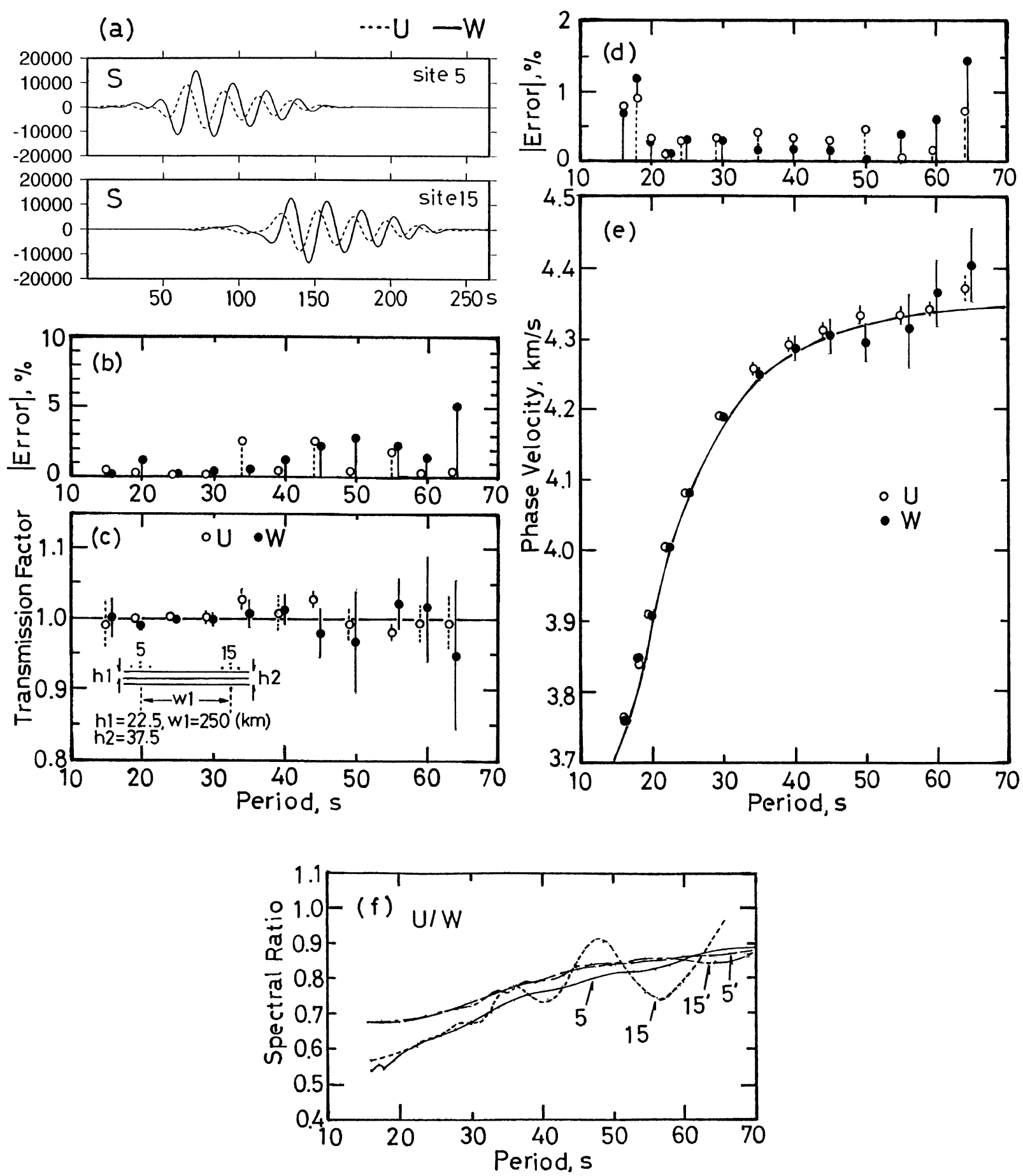

Fig. 2. (a) An example of Rayleigh waves of vertical (W) and radial (U) components. The waves at sites 5 and 15 are the signals recorded at the edges of the root at the wave's respective approach and retreat. (b) Absolute errors of spectral amplitudes of W- and U-components in terms of percent. The errors are plotted based on (c). (c) Average transmission factors of amplitudes of W- and U-components obtained using three pairs of seismograms recorded at sites 5 and 15 and in the vicinity of these sites (dots); a distance ( $w 1)$ of $250 \mathrm{~km}$ is maintained between the two sites. The distance between each dot is $25 \mathrm{~km}$. The seismograms are calculated for a stratified medium (Model S) with a crustal thickness of $h 2=37.5 \mathrm{~km}$, where the thickness ( $h 1$ ) of the upper crust is $22.5 \mathrm{~km}$. (d) Absolute errors of phase velocities of Rayleigh waves of W- and U-components. The errors are plotted based on (e). (e) Average phase velocities of Rayleigh waves of W- and U-components obtained using seismograms given in (c). Phase velocities of Wand U-components are shown by solid and open circles, respectively. Standard errors are indicated by vertical bars. Phase velocities calculated by the normal mode theory are indicated by a solid line. (f) Spectral ratios of U/W at sites $(5,15)$ and sites $\left(5^{\prime}, 15^{\prime}\right)$. The ratios at sites 5 and 15 are obtained from synthetic seismograms, those at sites 5' and 15' from normal mode solutions for spheroidal oscillations. Sites 5' and 15' correspond to sites 5 and 15 in the $x-z$ coordinates. 
dyne.cm. The waves correspond to the fundamental mode Rayleigh waves excited at a point with a focal depth of 10.3 $\mathrm{km}$, at the azimuth of 90 degrees as measured counterclockwise from the fault strike, with the shortest epicentral distance of $100 \mathrm{~km}$ (left edge of the computational space), and with a travel time of $92.21 \mathrm{~s}$ (the time $t=0$ in Eqs. (8), (9)). The entire seismogram $w$ or $u$ in the heterogeneous media consists of two parts, primary waves $w^{0}$ or $u^{0}$ and scattered waves $w^{1}$ or $u^{1}$ (Aki and Richard, 1980):

$$
\begin{gathered}
w(t, x, z)=w^{0}(t, x, z)+w^{1}(t, x, z) \\
u(t, x, z)=u^{0}(t, x, z)+u^{1}(t, x, z) .
\end{gathered}
$$

The primary waves would constitute the whole seismogram if the heterogeneity were absent. The scattered waves are generated by the interaction between primary waves and heterogeneities in the structure. Based on (10) and (11), the scattered waves $w^{1}$ or $u^{1}$ can be computed if the entire seismograms $w$ or $u$ and the primary waves $w^{0}$ or $u^{0}$ are obtained.

\section{Precision of the Numerical Results}

In the wave propagation in numerical wave motion fields, we encounter computation errors depending on the discretization of the medium and the physical parameters in the medium. The accuracy of amplitude and phase velocity dispersion is shown in Fig. 2, along with an example of incident (site 5) and transmitted (site 15) Rayleigh waves. Sites 5 and 15 correspond to the edges of the dent at the wave's respective approach and retreat. First, the synthetic seismograms of $\mathrm{W}$ - and U-components recorded at sites 5 and 15 were calculated for the stratified medium (Model S) for wave incidence from the left. The distance $(w 1=250$ $\mathrm{km}$ ) between sites 5 and 15, and the thicknesses of the upper $(h 1=22.5 \mathrm{~km})$ and lower $(h 2=37.5 \mathrm{~km})$ crusts are indicated in the figure (see also Fig. 1). Second, transmission factors were calculated by moving both station sites 25 $\mathrm{km}$ towards the right, and $25 \mathrm{~km}$ to the left, respectively; a distance of $250 \mathrm{~km}$ is maintained between the two sites. The transmission factor is defined as the amplitude spectrum at a station near site 15 normalized by the spectrum at a station near site 5 . Theoretically, the transmission factor should be unity, because a 2-D wave field is under consideration. Average transmission factors and standard errors are shown in the figure, indicating that standard errors of the U-component are less than $3 \%$ for periods of $15-65 \mathrm{~s}$. The standard errors for the $\mathrm{W}$-component are also less than $3 \%$ for periods less than $50 \mathrm{~s}$, though they become a little larger for periods longer than $50 \mathrm{~s}$. The absolute errors, estimated from the difference between transmission factors and unity, for both $\mathrm{W}$ - and U-components are less than 3\% for periods of $15-65 \mathrm{~s}$. On the whole, the accuracy of the amplitudes at long periods appears to be higher for the U-component than for the $\mathrm{W}$-component. This might be explained by the fact that the energy of incident waves at long periods decreases more gently for the U-component than for the $\mathrm{W}$-component (see Fig. 8(a)).

The accuracy of the phase information is determined by the phase velocity. The phase velocities were calculated, as well as performed for the transmission factors, by moving both stations (sites 5 and 15) $25 \mathrm{~km}$ towards the right, and $25 \mathrm{~km}$ to the left, respectively, so that a distance of $250 \mathrm{~km}$ is maintained between the two sites. A two-station method (Sato, 1978, pp. 252), which calculates differences in phase spectra between two stations, was used for the determination of average phase velocities between the two sites. Average phase velocities obtained using three pairs of seismograms recorded at sites 5 and 15 and in their vicinities are shown in Fig. 2(e). This figure indicates that the standard errors for the $\mathrm{W}$-component amount to almost less than $1.2 \%$ for periods of 15-65 s; the absolute errors (Fig. 2(d)), which also amount to less than $1.5 \%$ in the same period range, were estimated from the difference of average phase velocities determined above from a normal mode solution. The standard errors and absolute errors for the U-component, which are less than $0.5 \%$ and $1.0 \%$ for periods of $15-65 \mathrm{~s}$, respectively, are smaller than those for the $\mathrm{W}$-component. These results show that the accuracy of the phase velocities is higher for the U-component than for the $\mathrm{W}$-component, as was seen in the accuracy of amplitudes.

The characteristics of spectral ratios U/W are shown in Fig. 2(f). The ratios estimated from synthetic seismograms at sites 5 and 15 increase from 0.55 to 0.90 with an increase in period from 15 to $70 \mathrm{~s}$, whereas those predicted by normal mode solutions for spheroidal oscillations at sites 5' and 15' increase from 0.65 to 0.90 in the same period range. The epicentral distances of 600 and $850 \mathrm{~km}$, respectively, at sites 5 ' and 15', measured from the seismic source used for the calculation of initial values, correspond to those at sites 5 and 15 in the $x-z$ coordinates. On the whole, the ratios U/W for the simulations have similar characteristics to those for the normal mode solutions. A little higher ratios for the normal mode solutions than those for the simulations near $20 \mathrm{~s}$ might be caused by the performance of the simulations using Cartesian coordinates, whereas the normal mode solutions are obtained using spherical polar coordinates. The pertur-

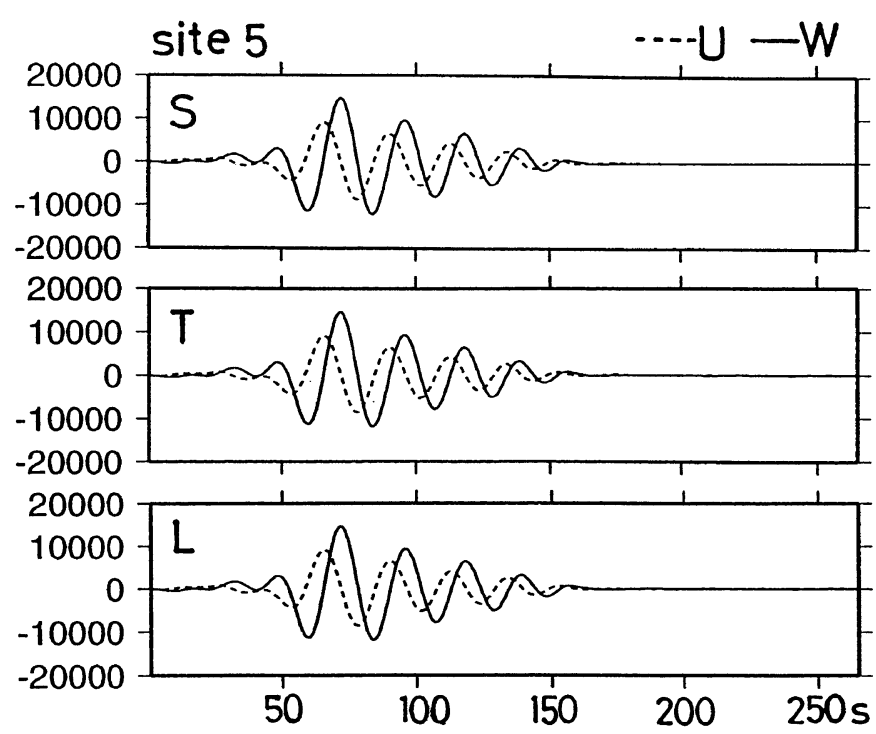

Fig. 3. Rayleigh waves of vertical (W) and radial (U) components for Models S, T, and L at site 5 . 
(S)

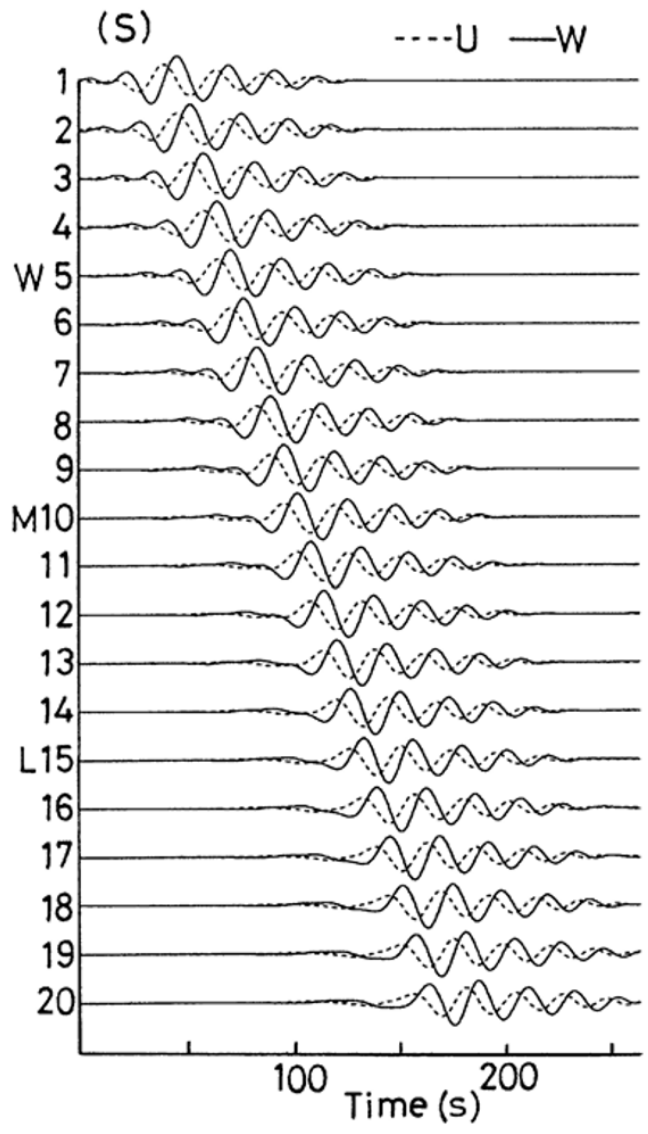

( $(T)$

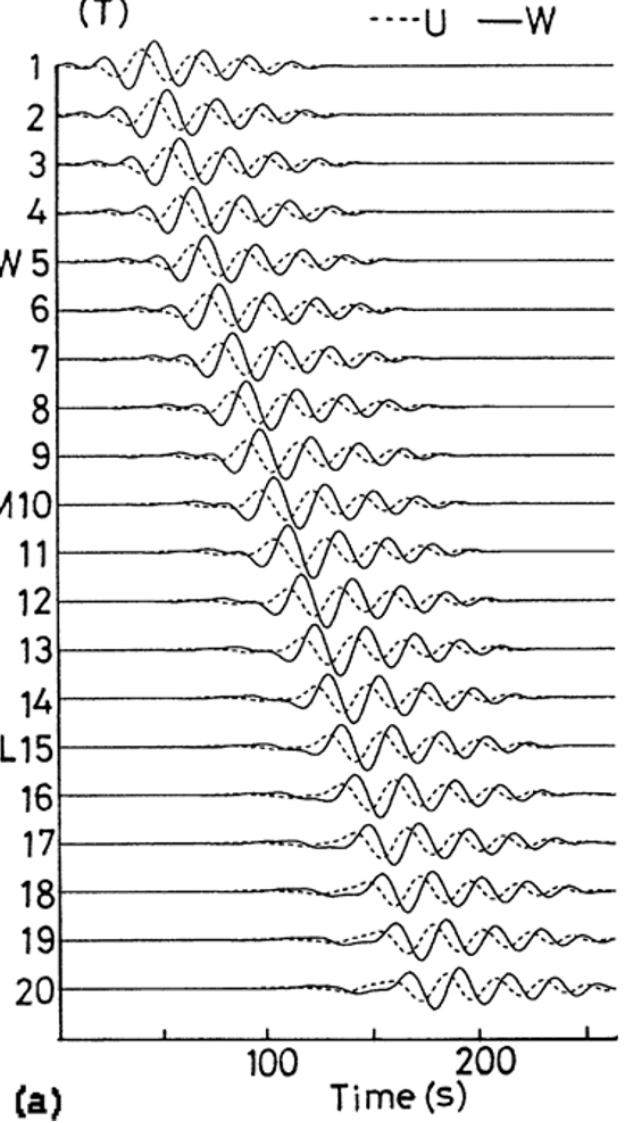

Vertical comp. $\quad \cdots \cdot(S)-(T)$

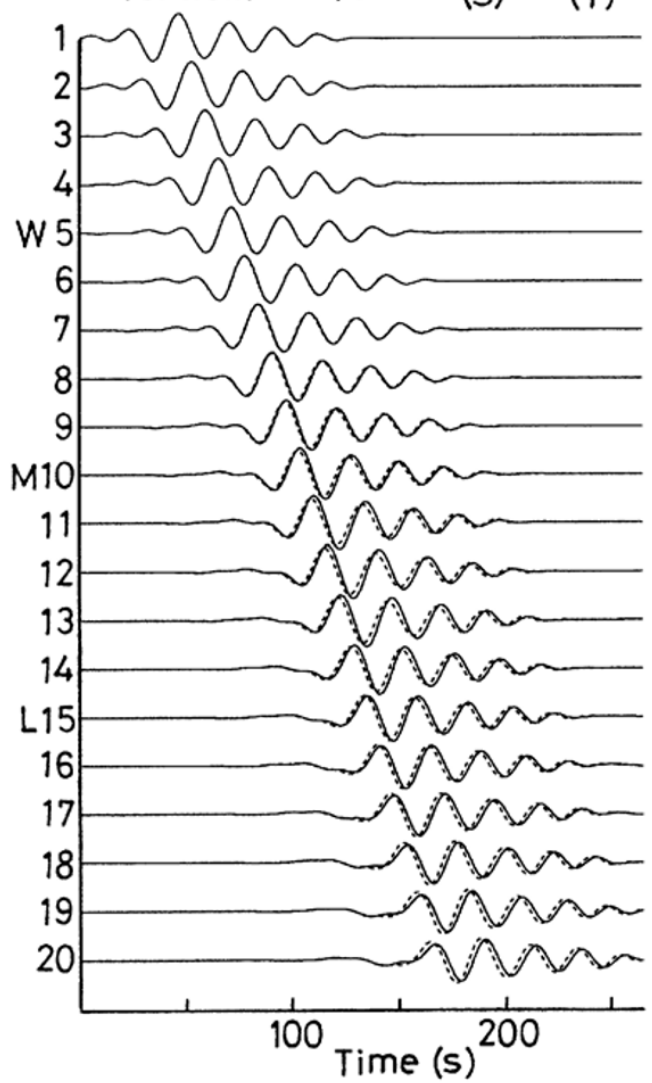

Radial comp.

$\cdots(\mathrm{S})-(\mathrm{T})$

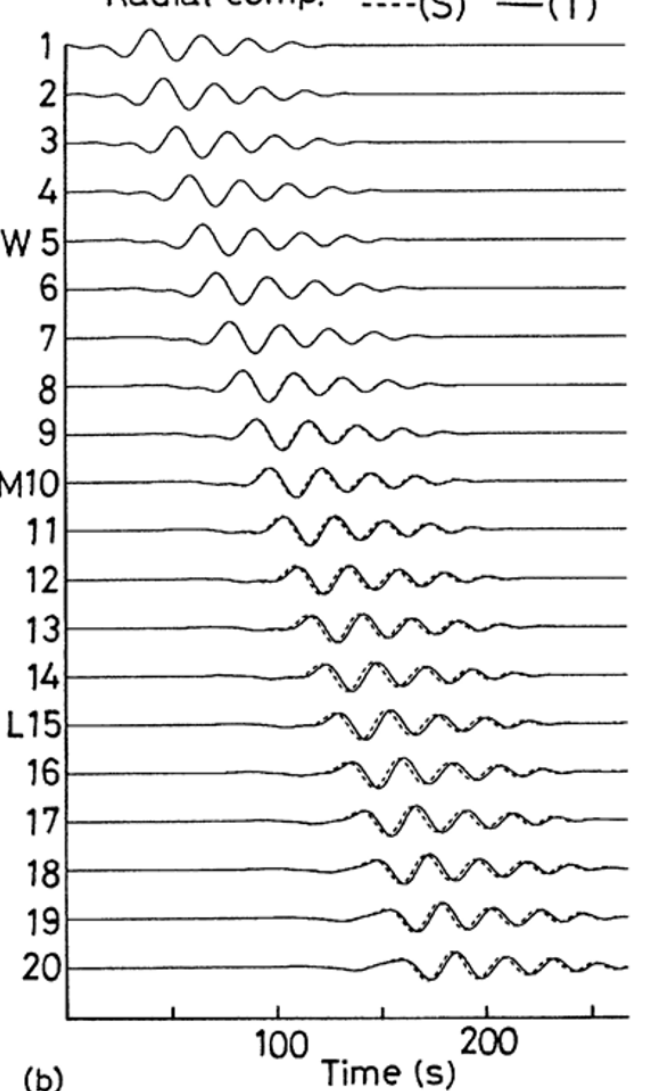

Fig. 4. (a) Computed seismograms of Rayleigh waves of vertical (W) and radial (U) components for various sites along the surface for Models $\mathrm{S}$ and T. The symbols W, M, and L next to the site numbers on the ordinate indicate, respectively, the windward, midpoint, and leeward locations over the mountain root. (b) Rayleigh waves of $\mathrm{W}$ - and U-components for various sites along the surface. The waves for Models $\mathrm{S}$ and $\mathrm{T}$ are traced by dotted and solid lines, respectively. The symbols $\mathrm{W}, \mathrm{M}$, and L are defined in the caption in (a). 

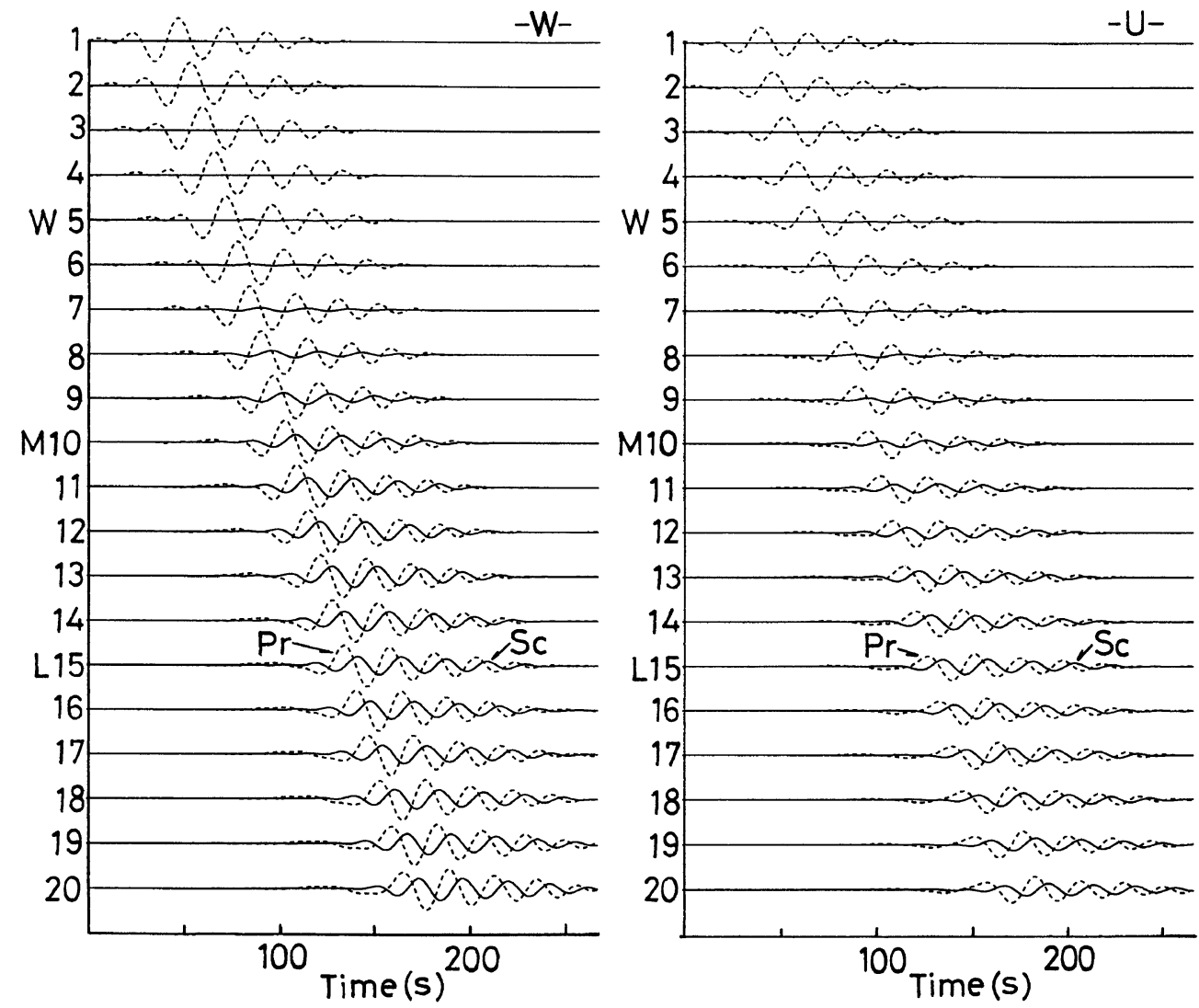

Fig. 5. Time series of scattered waves Sc (bold lines) and primary waves Pr (dotted lines) of W- and U-components for Model T. For the numerals and symbols W, M, and L attached to the wave forms, see the caption of Fig. 4.

bation of the ratios at site 15 is caused by the difference in accuracy between amplitudes of the $\mathrm{W}$ - and U-components, which were analyzed previously.

\section{Results and Discussion}

Incident Rayleigh waves recorded at site 5 for a stratified medium (Model S), a mountain root (Model T), and a mountain root with a low velocity zone LVZ (Model L) are shown in Fig. 3. Strictly speaking, the wave forms for Models T and $\mathrm{L}$ are different from those for Model $\mathrm{S}$ because the scattered waves, which were generated inside the mountain root, are superimposed on the waves for Models T and L.

\subsection{Simulated Rayleigh waves}

Examples of dispersed wave trains for Models $\mathrm{S}$ and $\mathrm{T}$, recorded at twenty sites, are shown in Fig. 4. Site numbers are sequentially denoted from left to right along the mountain root. The distance between two sites is fixed to be 25 $\mathrm{km}$. The discrepancy in phase lag between the wave trains for Models S and T can be observed in the figure. The phase lag between the two models cannot be clearly observed at site 5 , though it can be seen that the wave trains for Model $\mathrm{T}$ at site 10 , a midpoint of the mountain root, are plainly delayed relative to those for Model S. The waves of the Wand U-components for Model T given in Fig. 4, which will be defined as the entire waves (En) through Eqs. (10) and (11), consist of the primary (Pr) and scattered (Sc) waves. The high amplitudes of the scattered waves, shown in Fig. 5, are due mainly to the time delay between the entire waves and the primary waves. So the difference between them is high. To interpret the scattered waves, the delay across the structure must be taken into account, possibly using a coupled mode technique (e.g., Maupin and Kennett, 1987), but this is somewhat beyond the scope of the present paper. The snapshots (Fig. 6) of the entire and scattered waves of the $\mathrm{W}$ - and U-components show that the scattered waves appear after the passage of site 5 near a time of $100 \mathrm{~s}$ for depths of $H=0$ and $55 \mathrm{~km}$ and that the wavetrains for $H=55 \mathrm{~km}$ are decreased in short wavelength components, compared to those for $H=0 \mathrm{~km}$. As far as we see the scattered waves in Figs. 5 and 6, it seems that converted waves from fundamental to higher mode Rayleigh waves, whose velocities are higher than those of the fundamental mode Rayleigh waves, and reflected waves are not appreciable. The above circumstance can also be understood from the snapshots (Fig. 7) of the scattered waves for $H=0$ and $55 \mathrm{~km}$. In the region between the left edge $\mathrm{L}$ and site 5 , wavetrains such as converted or reflected waves are not detectable. Fundamental to higher mode Rayleigh wave conversion, reflections of Rayleigh waves, and Rayleigh to body wave conversion (Momoi, 1987) are likely to occur at boundaries with strong heterogeneities between uniform and nonuniform wave guides. In Love wave propagation (Yoshida, 1996), about $10 \%$ of the energy of incident waves at maximum is estimated to convert to body waves at periods near 20 or $50 \mathrm{~s}$ in the media with a step, in which the continental crustal thickness varies from 35 to $52.5 \mathrm{~km}$ and 


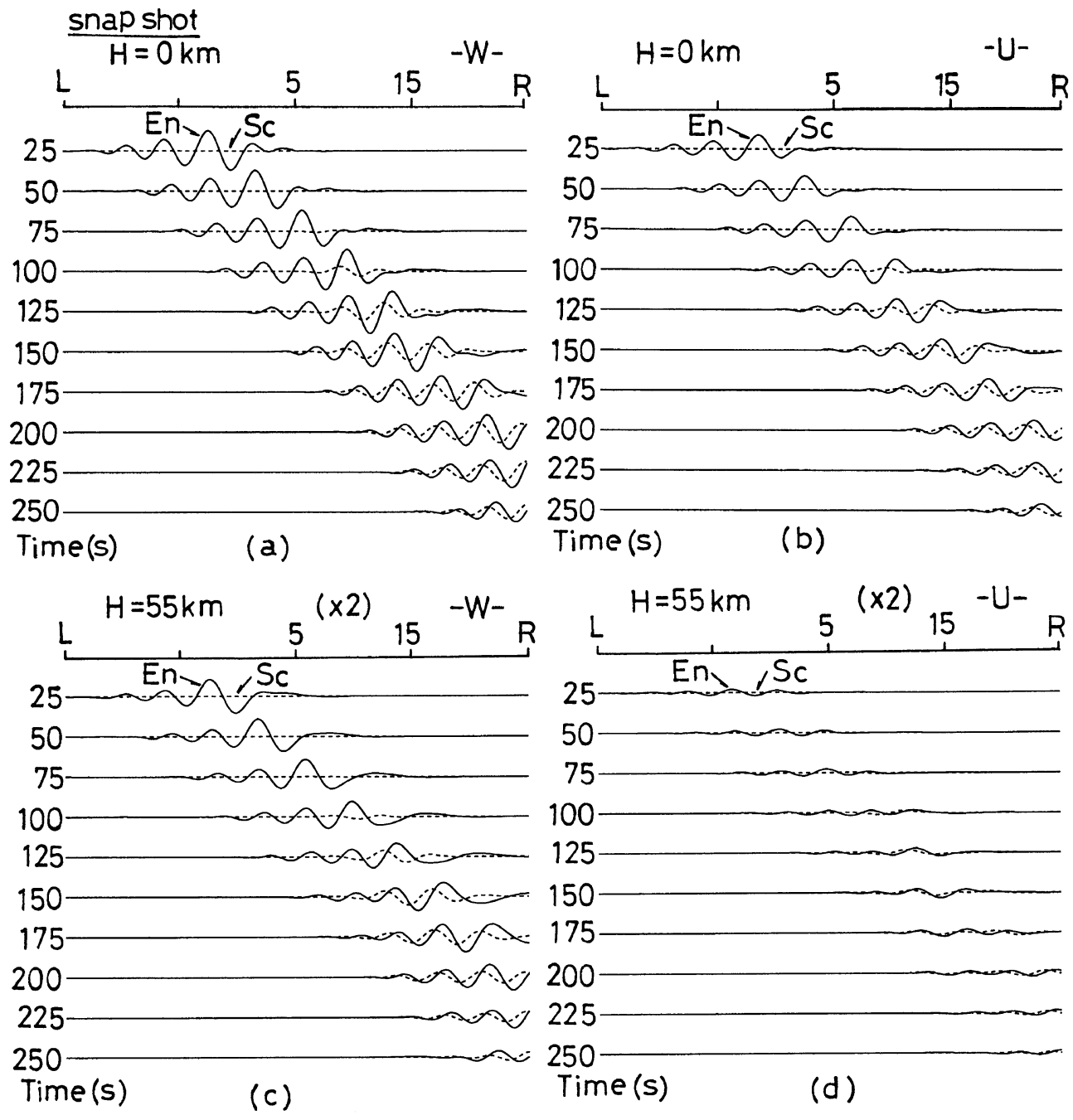

Fig. 6. Snapshots of the entire seismograms En (bold lines) and scattered waves Sc (dotted lines) of W- and U-components for Model T. Snapshots at depths of $H=0$ and $55 \mathrm{~km}$ are shown at the top (a,b) and bottom (c, d) in the figure, respectively. The amplitudes of the entire and scattered waves at $H=55 \mathrm{~km}$ are doubled. Symbols L, 5, 15, and R denoted on the abscissa correspond to those given in Fig. 1.

vice versa; if the slope of the crustal layers decreases, the wave conversion decreases. In Rayleigh wave propagation (Levander, 1985), the degree of scattering decreases to a half of that in Love wave propagation. In the present Tien Shan model the sloping angle of the upper and lower crustal layers is 8.5 degrees. So, a small quantity of the energy of incident Rayleigh waves might be consumed for conversion to body waves although it is not appreciable as seen in the snapshots shown above.

\subsection{Response characteristics}

Amplitude spectra of the waves at sites 5 and 15 for Models $\mathrm{S}, \mathrm{T}$, and L are shown in Fig. 8, along with the wave forms at site 15. The amplitude spectra of incident waves for Model S at site 5 show that the ratio of U/W (Fig. 8(a)) increases from 0.55 to 1.0 with an increase in period from 15 to $100 \mathrm{~s}$, respectively. The ratios of U/W (Fig. 8(b)) for the transmitted waves at site 15 (Fig. 8(c)) for the three models also show similar characteristics to those at site 5. However, the ratios of $\mathrm{U} / \mathrm{W}$ for Models $\mathrm{T}$ and $\mathrm{L}$ are significantly different from those for Model S at periods of 25-40 s. Namely, the former two ratios are lower than the last. This differ- ence results from a characteristic oscillation of the mountain root (Fig. 8(d)). The ratio of $\mathrm{W} / \mathrm{W}$ shows that the mountain root structure preferentially amplifies the waves for periods of $20-50 \mathrm{~s}$, with a maximum of about $7 \%$ near $30 \mathrm{~s}$, whereas the ratio of $\mathrm{U} / \mathrm{U}$ shows a monotonous increase in period, with a maximum of about $4 \%$ near $90 \mathrm{~s}$. The ratios $\mathrm{W} / \mathrm{W}$ and $\mathrm{U} / \mathrm{U}$ featured above can be interpreted to be the response characteristics of the mountain root structure. We also note differences in the ratios of W/W between Models $\mathrm{T}$ and L; the characteristics of the amplitude spectra of the Wcomponent for Model L are slightly shifted towards longer periods, compared to those for Model T, although distinct differences in the ratios of $\mathrm{U} / \mathrm{U}$ between the two models are not observed for the U-component.

\subsection{Effects of the LVZ}

Spectral characteristics depend on the S-wave velocity, size, and location of the LVZ. So, several simulations were performed for crustal and upper mantle models with a LVZ different from Model L. Cotton and Avouac (1994) presented a regional model for the eastern Tien Shan from surface wave group velocity data. A pronounced LVZ in the 


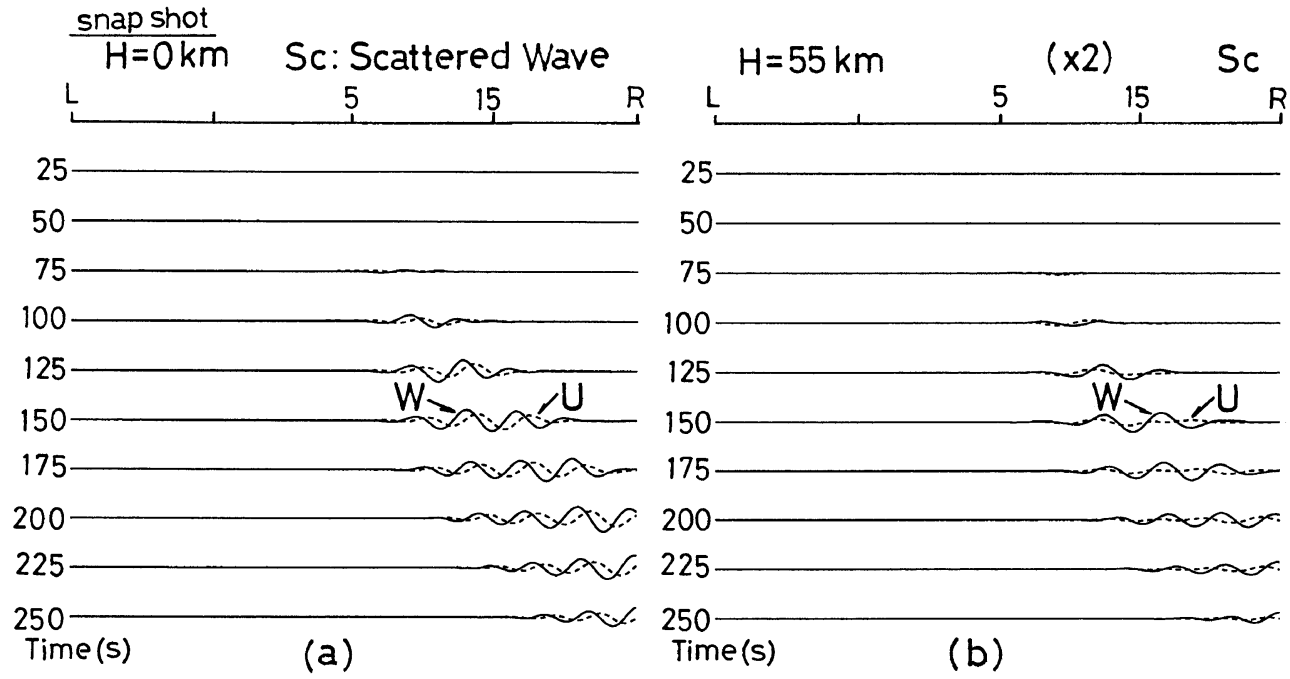

Fig. 7. Snapshots of the scattered waves of W- (bold lines) and U- (dotted lines) components at depths of $H=0$ and $55 \mathrm{~km}$ for Model T. The amplitudes of the waves at $H=55 \mathrm{~km}$ are doubled.

(a) Stratified Medium: $\mathrm{S}$

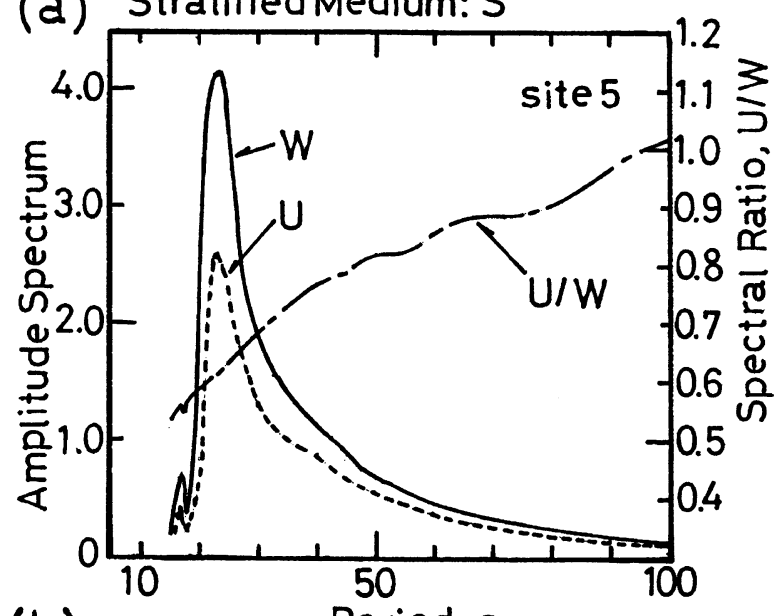

(b)

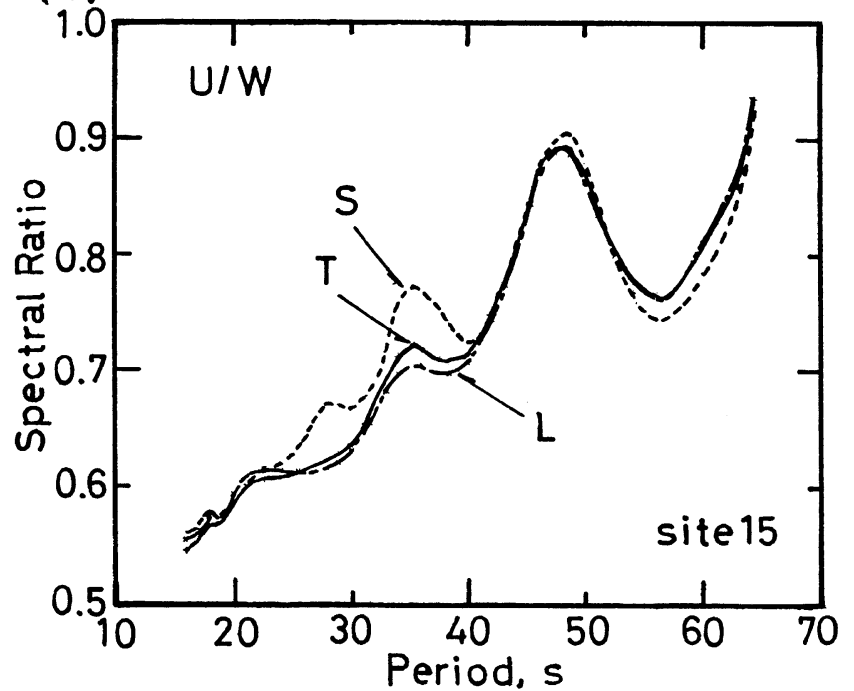

(c)

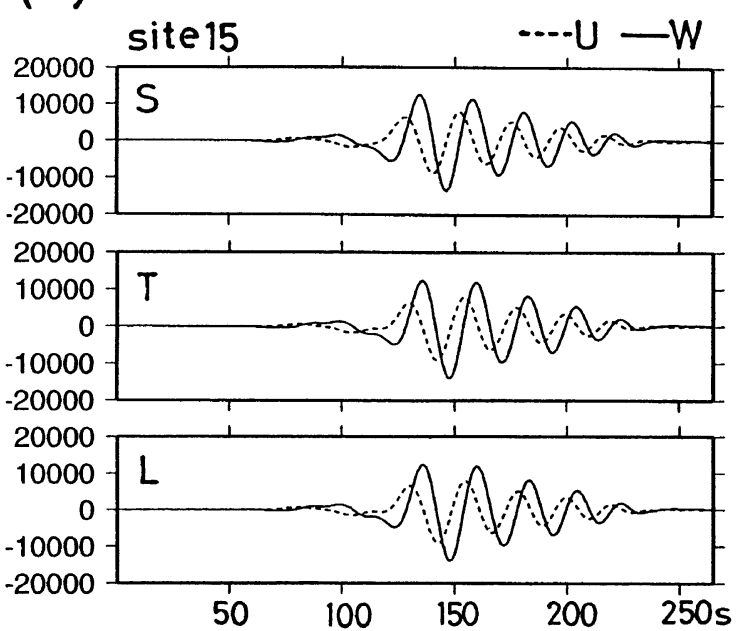

(d)

site 15

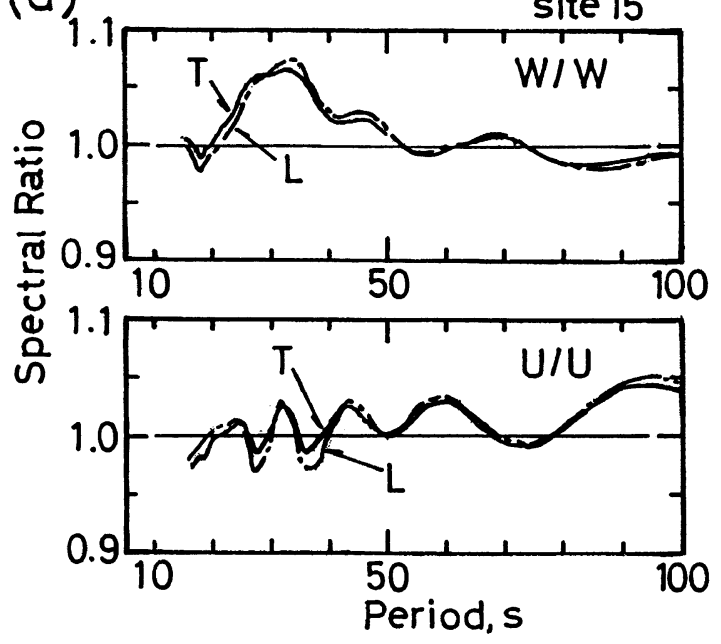

Fig. 8. (a) Amplitude spectra of Rayleigh waves of vertical (W) and radial (U) components and the ratios of U/W at site 5 for Model S. (b) Spectral ratios of U/W at site 15 for Models S, T, and L. (c) Rayleigh waves of W- and U-components at site 15 for Models S, T, and L. (d) Spectral ratios of W/W and $\mathrm{U} / \mathrm{U}$ at site 15 for Model T and Model L to that for Model S. 
lower crust and a second LVZ at depths below $100 \mathrm{~km}$, with S-wave velocities around 3.3 and $4.3 \mathrm{~km} / \mathrm{s}$, respectively, are involved in their model. In order to verify the effects of the LVZ on the amplitude spectra of the $\mathrm{W}$ - and U-components, several crustal and upper mantle models based on Model $\mathrm{L}$ were constructed and are shown in Fig. 9. Model LL has a second local LVZ with a horizontal length of $50 \mathrm{~km}$
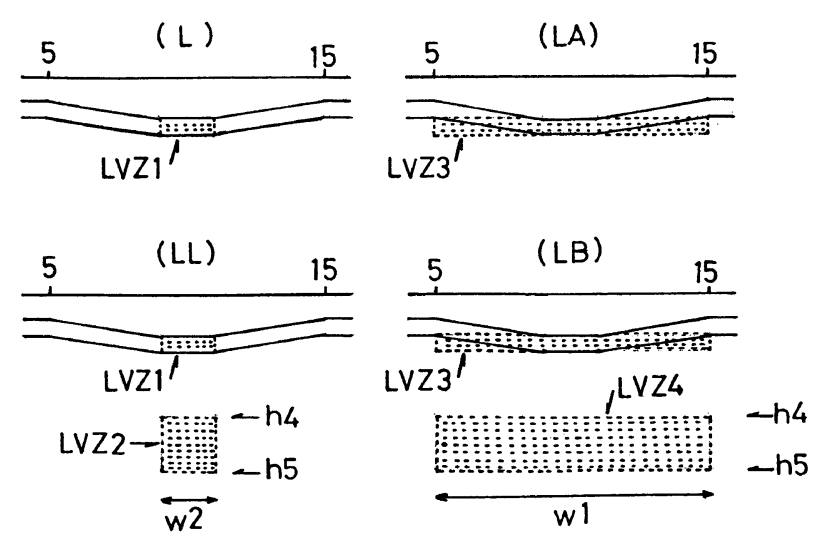

$\mathrm{h} 4=112.5 \mathrm{~km}$, $w 1=250 \mathrm{~km}$.
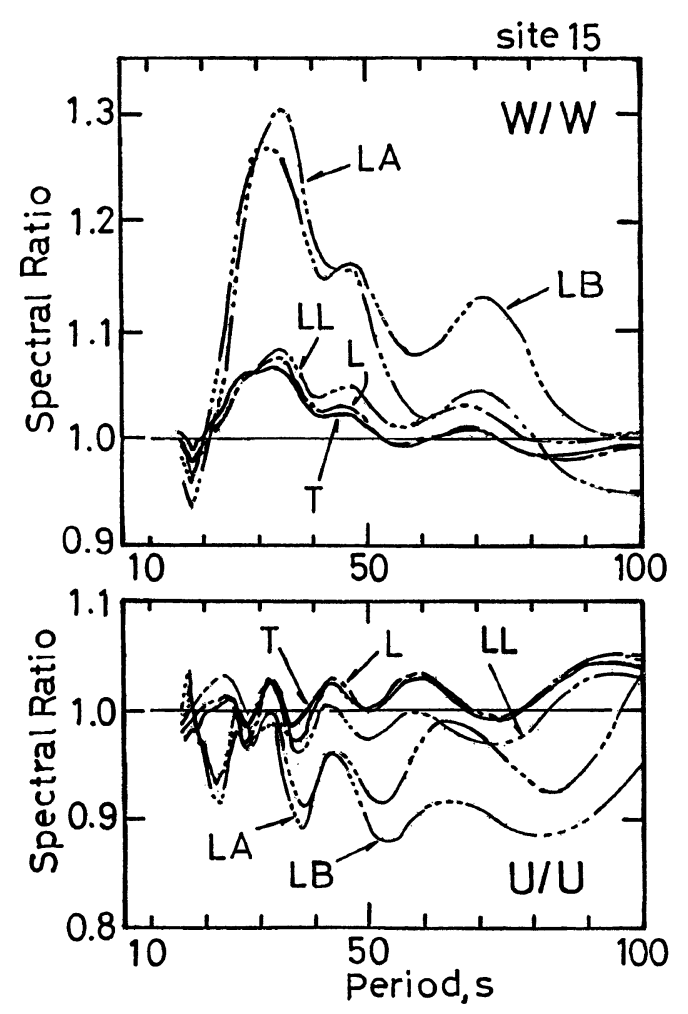

Fig. 9. Top: Schematic maps showing crustal and upper mantle structures involving one or a double low velocity zone (LVZ) beneath the mountain. Models L and LL have small-scale LVZs with a horizontal length of 50 $\mathrm{km}$. Model L has a LVZ in the lower crust whears Model LL has LVZs in both the lower crust and the upper mantle. Models LA and LB have a large-scale LVZ with a horizontal scale of $250 \mathrm{~km}$. Model LA has a LVZ in the lower crust whereas Model LB has LVZs in both the lower crust and the upper mantle. The location of the LVZ (dotted area) is indicated by LVZ1, LVZ2, LVZ3, and LVZ4. LVZ1 and LVZ3 exist at depths of $37.5-52.5 \mathrm{~km}$, LVZ2 and LVZ4 at depths of 112.5-162.5 km. Bottom: Spectral ratios of vertical $(\mathrm{W} / \mathrm{W})$ or horizontal $(\mathrm{U} / \mathrm{U})$ components for Models L, LL, LA, and LB to that for Model S. in the upper mantle at depths of about $110-160 \mathrm{~km}$. The LVZ with a horizontal length of $250 \mathrm{~km}$ for Models LA and LB is widely distributed under the mountain. For Model LA the LVZ exists merely in the lower crust whereas for Model LB it does in both the lower crust and the upper mantle. Physical parameters in the LVZ in the upper mantle, such as $V \mathrm{p}=7.3789 \mathrm{~km} / \mathrm{s}, V \mathrm{~s}=4.3433 \mathrm{~km} / \mathrm{s}$, and $\rho=3.1012 \mathrm{Mg} / \mathrm{m}^{3}$ (Cotton and Avouac, 1994), were used. For S-wave velocity in the lower crust, a $V \mathrm{~S}$ of $3.5 \mathrm{~km} / \mathrm{s}$ (Table 1) was used, which was assumed for Model L and is slightly higher than that reported by Cotton and Avouac (1994). Synthetic seismograms were calculated for these models for a Rayleigh wave incidence from the left, and the amplitude spectra at site 15 are shown in Fig. 9. We can clearly see in the figure the spectral shift of the Wcomponent for Models T and LL, mentioned previously for Models $\mathrm{T}$ and $\mathrm{L}$. This phenomenon suggests that the existence of the small-scale LVZ beneath the mountain preferrentialy amplifies the waves of the $\mathrm{W}$-component at longer periods (20-50 s), in comparison with the case without the LVZ. These characteristics seem to be related to the simulation results of Pedersen et al. (1998, figure 15) who showed that the amplitude spectra of Love waves, which are converted from Rayleigh waves across the mountain root structure with the LVZ, are enriched at longer periods compared to those for the mountain root structure without the LVZ. When the LVZ is widely distributed beneath the mountain (Models LA and LB), the amplitudes of the W-component are extensively amplified for periods of 20-60 s. The existence of a second LVZ in the upper mantle (Model LB) strongly amplifies the amplitudes for periods longer than $50 \mathrm{~s}$ in comparison with the case which has a LVZ merely in the lower crust (Model LA). On the contrary, the spectra of the U-component show a result opposit to those of the Wcomponent. Namely, the amplitudes decrease in accordance with the increase of the LVZ. This might be explained by the property that Rayleigh waves of the $\mathrm{W}$ - and U-components are connected with $\mathrm{P}$ - and SV-waves; S-waves are likely to attenuate when the LVZ is involved in the ray path. The particle orbits of the U-component are parallel to the horizontally elongated LVZ.

\subsection{Rayleigh to Love wave conversion}

In order to evaluate the effects of the LVZ on Rayleigh to Love wave conversion, a numerical experiment regarding an oblique incidence of Rayleigh waves was implemented. The simulations were performed for a Rayleigh wave incidence upon the front (site 5) of the mountain with an azimuthal angle of incidence of 30 degrees, in which the mountain root structure shown in Fig. 1 was assumed to be extended in the transverse direction. Initial values similar to those described previously was used and a time increment of $\Delta t=0.3 \mathrm{~s}$ was assumed. Synthetic seismograms were calculated at the edge ( site 15") of the mountain at the wave's retreat, employing the 3-D finite difference scheme (Mikumo et al., 1987; Frankel and Vidale, 1992; Yoshida, 1996, 2000). The distance between sites 5 and 15 " has a length of $290 \mathrm{~km}$. The amplitude spectra of the W-, U-, and V (transverse)components at site 15", calculated for Models T and LB, are shown in Fig. 10. This figure shows that the amplitudes of the V-component for Model LB are extensively dominated 


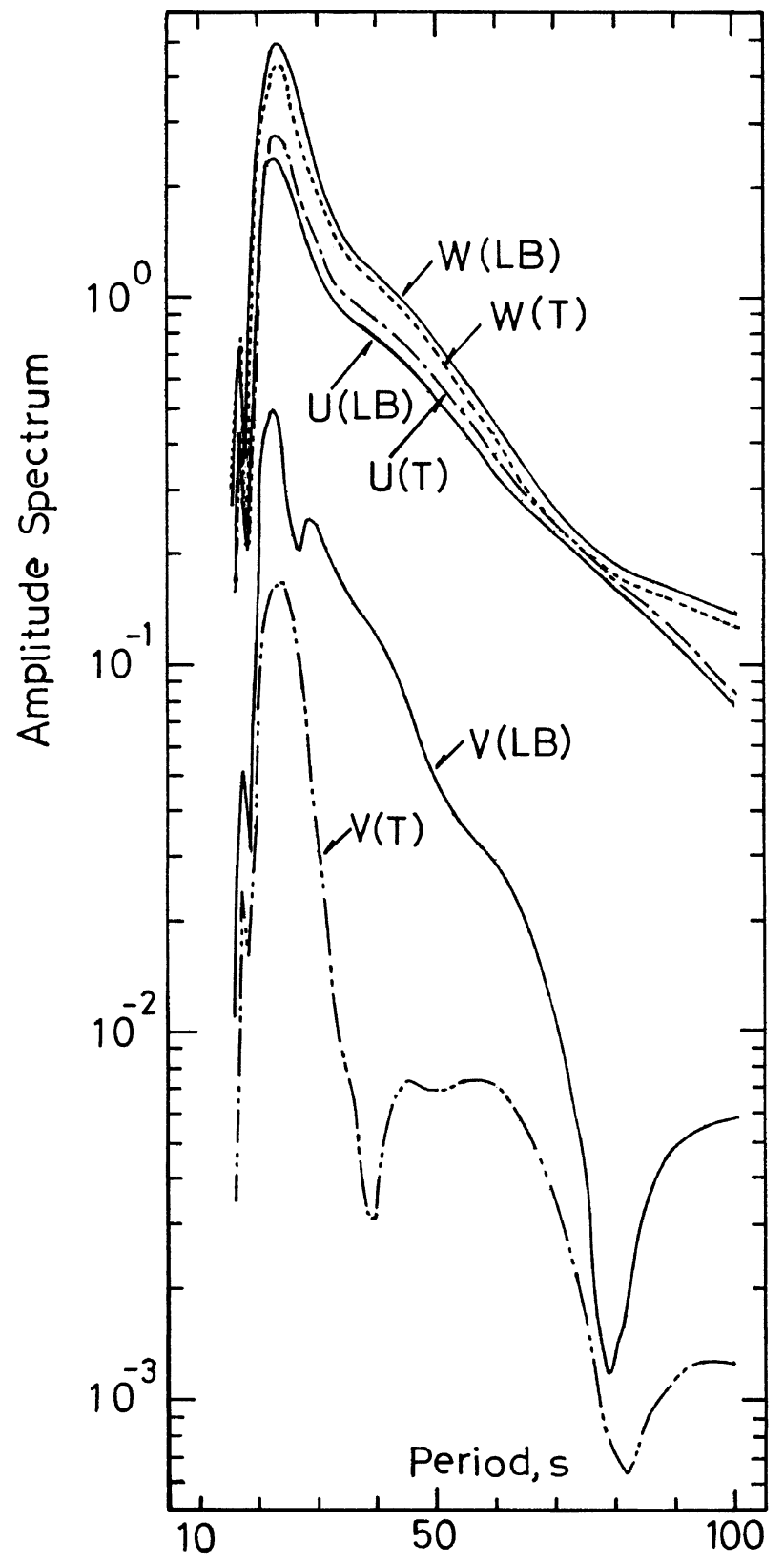

Fig. 10. Amplitude spectra of the waves of W-, U-, and V-components recorded at the edge of the mountain at the wave's retreat for an oblique incidence of Rayleigh waves. The waves are calculated with the azimuthal angle of incidence of 30 degrees for Models T and LB. Model names are denoted in the parentheses. The mountain root structure shown in Fig. 1 is assumed to be extended in the transverse direction and the waves are obliquely incident on the front side (site 5) of the mountain.

for periods of 20-70 s, whereas those for Model $\mathrm{T}$ are predominant for periods of 20-40 s. These results indicate that the existence of the LVZ in the lower crust and the upper mantle converts Rayleigh to Love waves stronger than the case without the LVZ over a wide period, as well as observed in the spectra of the W-component for Model LB (Fig. 9).

\section{Applicability of Mountain Root Models}

The Tien Shan mountain is located in northwest of the Chinese test site at Lop Nor. Surface waves observed at stations of the OBN (Russia) and the FLN (France) resulting from nuclear explosions at Lop Nor traverse the Tien Shan range. By analyzing observed surface waves from Lop Nor, Pedersen et al. (1998, figure 3) quantified high Love wave amplitudes in the spectral domain in a frequency interval between 0.015 and $0.05 \mathrm{~Hz}$. By implementing numerical simulations of surface waves for a medium locally approximated by a 2-D structure, they speculated that the observed Love waves are created by conversion of Rayleigh waves in the Tien Shan mountain. The simulations were performed using the indirect boundary element method, and showed that spectral amplitudes of the transverse (V) component increase with an increase in angle (10-50 deg.) of incidence of Rayleigh waves for frequencies of $0.02-0.1 \mathrm{~Hz}$.

The mountain root models of Models T and L (Fig. 1) are simple ones approximating the crustal structures of the Tien Shan mountain. Elastic parameters (Table 1) for the Tien Shan were adopted from Pedersen et al. (1998). The crustal thickness of the mountain root of $52.5 \mathrm{~km}$, assumed in Models $\mathrm{T}$ and $\mathrm{L}$, is close to those of 49 and $55 \mathrm{~km}$ used in their report. The horizontal width $(w 1=250 \mathrm{~km})$ of the mountain root for the above models is slightly narrower than the significant crustal thickening area of $300 \mathrm{~km}$ (Burov et al., 1990; Cotton and Avouac, 1994). However, the configurations of the Tien Shan mountain in the present models and in those used by Pedersen et al. (1998) are highly similar. It has been reported by Cotton and Avouac (1994) from observed Rayleigh wave dispersions that the regional models for the central and eastern Tien Shan have pronounced low-velocity layers in the crust and the upper mantle. The regional crustal and upper mantle structures were approximated by Models L, LL, LA, and LB. Thus, the spectral results obtained in the present work can be effectively compared with the results of their observations and simulations. We note that the frequency range of $0.02-0.05 \mathrm{~Hz}$, in which Rayleigh waves of the $\mathrm{W}$-component are greatly amplified at a maximum of 7\% for Models $\mathrm{T}$ and L, agrees with that of $0.015-0.05$ $\mathrm{Hz}$, in which the observed Love waves have predominant spectral amplitudes (Perdersen et al., 1998, figure 3b,c and figure $4(\mathrm{OBN}, \mathrm{FLN}))$. Taking into consideration the above observational results that the spectra of Love waves are predominant over a wide range beyond $50 \mathrm{~s}$, the LVZ seems to be widely distributed beneath the Tien Shan range in the lower crust and the upper mantle, as shown in the spectra of the V-component for Model LB (Figs. 9 and 10).

\section{Conclusion}

The spectra of Rayleigh waves across the mountain root, which approximates the Tien Shan mountain located in the northwest region of the Tarim basin in China, have been investigated using 2-D modeling analyses. The mountain root has a maximum crustal thickness of about $50 \mathrm{~km}$. Spectral amplitudes of $\mathrm{W}$ - and U-components show the following important characteristics: 1) Amplitudes of the W-component are strongly amplified for periods from 20 to $50 \mathrm{~s}$, with a maximum of about 7\% near 35 s. 2) Amplitudes of the Ucomponent show a slight increase with an increase in period. 3 ) For the mountain root structure with a double small-scale LVZ in the root and the upper mantle, amplitudes of the Wcomponent are enriched at slightly longer periods (20-50 s) relative to those for the mountain root structure without the 
LVZ. 4) For the mountain root structure with a double largescale LVZ in the lower crust and the upper mantle, at depths of $17.5-52.5 \mathrm{~km}$ and $112.5-162.5 \mathrm{~km}$, respectively, amplitudes of the $\mathrm{W}$-component are extensively amplified over a wide range beyond $50 \mathrm{~s}$; those of the U-component tend to decrease in the same period range. In a comparison with previously observed data (Pedersen et al., 1998), the following properties are notable: 5) The frequency range of $0.02-0.05 \mathrm{~Hz}$, featured in 1), is consistent with the range of $0.015-0.05 \mathrm{~Hz}$, in which the Rayleigh to Love wave conversion is dominant in observed surface waves across the Tien Shan mountains; 6) The spectra of observed Love waves, which are predominant over a wide range beyond $50 \mathrm{~s}$, can be more adequately explained by a mountain model involving the double large-scale LVZ beneath the mountain, with the horizontal scale of the LVZ comparable to that of the mountain root structure, rather than by a model without the LVZ.

Acknowledgments. Computations were performed at the earthquake information center, Earthquake Research Institute, and at the information technology center, University of Tokyo. The author thanks Fenglin Niu and Makoto Matsubara for their assistance with Generic Mapping Tools. The author is grateful to Helle Pedersen and Reiji Kobayashi for their useful comments.

\section{References}

Aki, K. and P. G. Richard, Quantitative seismology. Theory and method, Vol. II, 779-780, W. H. Freeman and Company, San Francisco, 1980.

Aki, K. and Y.-B. Tsai, Mechanism of Love-wave excitation by explosive sources, J. Geophys. Res., 77, 1452-1475, 1972.

Archambeau, C. B. and C. Sammis, Seismic radiation from explosions in prestressed media and the measurement of tectonic stress in the Earth, Rev. Geophys., 8, 473-499, 1970.

Bostock, M. G., Surface wave scattering from 3-D obstacles, Geophys. J. Int., 104, 351-370, 1991.

Burov, E. V., M. G. Kogan, H. Lyon-Caen, and P. Molnar, Gravity anomalies, the deep structure, and dynamic processes beneath the Tien Shan, Earth Planet, Sci. Lett., 96, 367-383, 1990.

Clayton, R. and B. Engquist, Absorbing boundary conditions for acoustic and elastic wave propagations, Bull. Seis. Soc. Am., 67, 1529-1540, 1977.

Cotton, F. and J. P. Avouac, Crustal and upper-mantle structure under the Tien Shan from surface-wave dispersion, Phys. Earth Planet. Inter., 84, 95-109, 1994.

Frankel, A. and J. Vidale, A three-dimensional simulation of seismic waves in the Santa Clara Valley, California, from a Loma Prieta aftershock, Bull. Seis. Soc. Am., 82, 2045-2074, 1992.

Friederich, W., E. Wielandt, and S. Stange, Multiple forward scattering of surface waves: comparison with an exact solution and Born singlescattering methods, Geophys. J. Int., 112, 264-275, 1993.

Harkrider, D. G., J. L. Stevens, and C. B. Archambeau, Theoretical Rayleigh and Love waves from an explosion in prestressed source regions, Bull. Seis. Soc. Am., 84, 1410-1442, 1994.

Kennett, B. L. N., Guided wave propagation in laterally varying media-I. Theoretical developments, Geophys. J. R. Astr. Soc., 79, 235-255, 1984.

Kobayashi, R., I. Nakanishi, and S. Tsuboi, Polarization anomalies of surface waves recorded by a broadband seismometer network in Hokkaido, Japan, J. Phys. Earth, 45, 383-396, 1997.

Kosarev, G. L., N. V. Petersen, L. P. Vinnik, and S. W. Roecker, Receiver functions for the Tien Shan analog broadband networks: Contracts in the evolution of structures across the Talasso-Fergama faults, J. Geophys. Res., 98, 4437-4449, 1993.

Levander, A. R., Finite difference calculation of dispersive Rayleigh wave propagation, Tectonophys., 113, 1-30, 1985.

Levshin, A., L. Ratnikova, and J. Berger, Peculiarities of surface-wave propagation across central Eurasia, Bull. Seis. Soc. Am., 82, 2464-2493, 1992.

Masse, R. P., Review of seismic source models for underground nuclear explosions, Bull. Seis. Soc. Am., 71, 1249-1268, 1981.

Maupin, V. and B. L. N. Kennett, On the use of truncated modal expansions in laterally varying media, Geophys. J. R. Astr. Soc., 91, 837-851, 1987.

Mikumo, T., K. Hirahara, and T. Miyatake, Dynamical fault rapture process in heterogeneous media, Tectonophys., 144, 19-36, 1987.

Momoi, T., Scattering of Rayleigh waves by a semi-circular rough surface on layered media, Bull. Earthq. Res. Inst., Univ. Tokyo, 62, 163-200, 1987.

Pedersen, H. A., J. P. Avouac, and M. Campillo, Anomalous surface waves from Lop Nor nuclear explosions: Observations and numerical modeling, J. Geophys. Res., 103, 15,051-15,068, 1998.

Press, F. and C. B. Archambeau, Release of tectonic strain of underground nuclear explosions, J. Geophys. Res., 67, 337-343, 1962.

Saito, M., Excitation of free oscillations and surface waves by a point source in a vertically heterogeneous earth, J. Geophys. Res., 72, 36893699, 1967.

Sato, Y., Dansei Hadouron (Theory of elastic wave motions), pp. 252, Iwanami Shoten, 1978 (in Japanese).

Snieder, R., 3-D linearized scattering of surface waves and a formalism for surface wave holography, Geophys. J. R. Astr. Soc., 84, 581-605, 1986.

Tanimoto, T., Modelling curved surface wave paths: membrane surface wave synthesis, Geophys. J. Int., 102, 89-100, 1990.

Yoshida, M., Group velocities, wave forms, and particle orbits of the first higher mode of oceanic Rayleigh waves excited by a deep earthquake on October 7, 1966, New Hebrides Islands, J. Phys. Earth, 32, 113-136, 1984.

Yoshida, M., Study on the propagation of Love waves across irregular structures of the Moho discontinuity, Bull. Earthq. Res. Inst., Univ. Tokyo, 71, 103-226, 1996.

Yoshida, M., Fluctuation of group velocity of Love waves across a dent in the continental crust, Earth Planets Space, 52, 393-402, 2000.

M. Yoshida (e-mail: mitsuru@eri.u-tokyo.ac.jp) 